\title{
NiceTown - Um Jogo Eletrônico para Ensino da Educação Ambiental
}

\author{
Máverick A. Ferreira ${ }^{1}$, Higor M. Santos ${ }^{1}$, Edjanio Oliveira, ${ }^{1}$ Antônio M. Lucena ${ }^{1}$ \\ ${ }^{1}$ Universidade de Pernambuco (UPE) - Garanhuns, PE - Brasil \\ \{amaverick70, higormonteiro1206, edjanio.upe,tn.mariano\}@gmail.com
}

\begin{abstract}
Resumo: Os jogos eletrônicos educativos têm se tornado cada vez mais presente, como uma ferramenta lúdica, no processo de ensino $e$ aprendizagem. Este trabalho tem como objetivo apresentar o desenvolvimento $e$ avaliação do jogo NiceTown. O mesmo tem a proposta de ensinar dois temas da Educação Ambiental: Coleta Seletiva e Saneamento Básico. O jogo foi desenvolvido por estudantes de Licenciatura em Computação e contou com o apoio de professores de Psicologia da Aprendizagem e de Ciências. Por sua vez, ele foi avaliado pelo seu público-alvo caracterizado por alunos do $6^{\circ}$ Ano. Como resultado, o jogo foi avaliado de maneira bastante positiva pelos professores e pela grande maioria dos alunos que participaram do teste.
\end{abstract}

Abstract: Educational electronic games have become increasingly present, as a ludic tool, in teaching and learning process. This paper aims to present the development and evaluation of the game called NiceTown. This game has proposal to teach two topics of the Ambient Education: Waste Recycling and Sanitation. NiceTown was developed by students of Computer and had support of professors of Psychology Learning and Sciences. In turn, it was evaluated for its target audience characterized by students of 6th year of middle school. As a result, the game was very positively evaluated by teachers and most students who participated in the test.

\section{Introdução}

Uma das questões mais discutidas atualmente é a sustentabilidade ambiental, pois o ser humano com suas ações extremamente capitalistas tem afetado de forma direta o ecossistema do planeta. A veracidade dos impactos ambientais provocados pelo desenvolvimento dos aspectos culturais, sociais, econômicos, populacionais e industriais, torna a educação ambiental extremamente necessária. Nesse sentido, Melo (2009) sugere que as escolas devem promover ações educativas que tenham por finalidade a preservação ambiental, sendo abordados vários aspectos, tais como, poluição, saneamento básico e desmatamento.

Aos poucos, a educação ambiental tem se tornado realidade nas escolas brasileiras. Segundo pesquisa realizada pelo Ministério da Educação (2007) em conjunto com a UNESCO, em que 217 das 317 escolas pesquisadas têm como principal objetivo para a educação ambiental, conscientizar alunos e comunidade quanto à importância da natureza, bem como conviver com a mesma.

Diante dos problemas ambientais enfrentados atualmente e a importância da escola na formação de cidadãos, a inovação no âmbito escolar torna-se necessária. Desta forma, Gomes (2012) ressalta que os jogos eletrônicos educativos é uma das 
grandes possibilidades tecnológicas para o ambiente escolar até 2017 com a intenção de educar de forma lúdica em todas as áreas de conhecimento. Nesse contexto, os jogos eletrônicos educativos surgem como aliados no processo de ensino e aprendizagem (PIETRUCHINSKI, 2012). Importância também citada por Malaquias e Ribeiro (2013), os quais indicam que brincar é um impulso natural da criança, que aliado à aprendizagem, torna mais fácil a obtenção do aprender devido à espontaneidade das brincadeiras por meio de uma forma intensa e total.

Diante da importância e necessidade da conscientização ambiental no âmbito escolar e da facilidade de aprendizagem dos alunos em meio à ludicidade, o presente trabalho tem como principal objetivo relatar o desenvolvimento e a avaliação de um jogo eletrônico educativo, denominado NiceTown, com ênfase em Educação Ambiental. Desse modo, o trabalho está organizado da seguinte maneira: A Seção 2 aborda os métodos utilizados para desenvolver e avaliar o jogo. A Seção 3 apresenta os resultados e, por fim, a Seção 4 demonstra as considerações finais e também recomendações para trabalhos futuros.

\section{Métodos}

O jogo apresentado neste trabalho foi resultado da disciplina Estágio Supervisionado III do curso de Licenciatura em Computação da Universidade de Pernambuco. Esta disciplina tem como ênfase o desenvolvimento de softwares educativos. Nesse aspecto, os estudantes matriculados foram agrupados em três equipes e ao longo do semestre foram acompanhados por três professores da área de Computação. Cada professor ficou designado para orientar técnica e metodologicamente um grupo.

Inicialmente, foi apresentado pelos professores da disciplina a abordagem Project Model Canvas (PMC) para uma gestão visível e prática de projetos (REIS, 2014). De forma análoga ao Business Model Canvas, os fatores que o PMC aborda são: justificativas, produto, objetivos, benefícios, requisitos, pessoas envolvidas, equipe, premissas, riscos, grupo de entregas, linha do tempo e custos. Em seguida, os grupos fizeram uma pesquisa sobre os softwares a serem desenvolvidos e o PMC foi iterativamente sendo preenchido e atualizado. O tema escolhido pelos autores deste trabalho foi a Educação Ambiental devido à motivação descrita na seção anterior.

Após definir o tema, um professor de Ciências do Ensino Fundamental foi escolhido e acompanhou todo o processo de desenvolvimento, fornecendo orientações a respeito do domínio da aplicação. De acordo com o cronograma, em duas semanas o grupo desenvolvia o software sob a orientação de um professor e na terceira semana havia encontros gerais com toda turma para apresentar o que havia sido feito, dificuldades encontradas, status do software e pretensões futuras. Além dos três professores de Computação, participava também das apresentações gerais uma professora doutora em psicologia da aprendizagem. O intuito era ter um momento para fornecer feedbacks sobre os aspectos técnicos e pedagógicos.

Ao analisar a viabilidade de tempo, um semestre, e a disponibilidade da professora de Ciências, foi escolhido o processo de desenvolvimento Iterativo e Incremental. Segundo Pressman (2006), um sistema deve ser desenvolvido de forma incremental, em que cada incremento permite adicionar ao sistema novas capacidades funcionais até a obtenção do sistema final. Esse processo de desenvolvimento tem como objetivo apresentar um produto operacional a cada incremento realizado. Desta forma, a 
cada reunião com a professora de Ciências e a cada apresentação geral, uma versão funcional do jogo era apresentada e coletavam-se os feedbacks de melhoria. Durante o processo de desenvolvimento do jogo, levou-se em consideração alguns conceitos de usabilidade e da engenharia congnitiva, tais como: (1) percepção - utilizou-se o conceito de signos, facilitando o reconhecimento das opções pelos usuários; (2) memorização - foram utilizadas figuras para facilita a memorização; (3) atenção desenvolveu-se uma interface com o mínimo de informação possível, além de cores e animações atrativas.

Para desenvolvimento do jogo, foi utilizada a ferramenta gratuita GameMaker 8.1 da empresa YOYO GAMES ${ }^{1}$. Esta ferramenta permite a criação de jogos em duas dimensões e simula também jogos de três dimensões. Ela roda em Sistema Operacional Microsoft Windows 95 ou superior (GOMES, 2006). O GameMaker disponibiliza uma gama de ferramentas necessárias para o desenvolvimento das funcionalidades do jogo, como também permite a implementação de códigos através de uma linguagem de programação própria, denominada GML (Game Maker Language).

Este trabalho foi realizado na Escola de Aplicação Professora Ivonita Alves Guerra, a qual foi escolhida por conveniência pelo fato de situar-se no mesmo prédio da Universidade. O jogo foi aplicado na disciplina de Ciências em uma turma do $6^{\circ}$ ano, tendo em vista, que os conteúdos abordados no jogo são também trabalhados nessa disciplina. A turma, escolhida pelo professor de Ciências, tinha 40 alunos. O jogo foi aplicado individualmente, não sendo permitida a utilização de materiais de apoio. Por fim, os alunos responderam a um questionário adaptado e baseado no modelo proposto por Savi et al. (2010).

Diante do potencial dos jogos para o processo de ensino e aprendizagem, é preciso atentar para a importância de avaliar tal ferramenta, de modo a identificar sua contribuição para a aprendizagem e desenvolvimento cognitivo dos alunos. O modelo de avaliação proposto por Savi et al. (2010), leva em consideração métodos de avaliação de jogos verificados na literatura como eficazes e confiáveis (KIRKPATRICK, 1994; KELLER e Susuki, 2009; BLOOM, 1956). O modelo de Savi busca avaliar a percepção dos alunos em relação a experiência de aprendizagem, conforme o Nível 1 de Kirkpatrick (1994), ao utilizarem um determinado jogo educacional. No entanto, o modelo de kirkpatrick deixa em aberto os parâmetros que devem ser avaliados.

Considerando esta proposta de se avaliar um jogo através da percepção que os alunos tiveram, foram incluídos nesta estrutura o modelo ARCS para avaliação do nível de motivação, componentes de User Experience em jogos para avaliação da experiência de interação com o jogo e princípios da taxonomia de Bloom para avaliação do impacto na aprendizagem do aluno. Para uma melhor contextualização do modelo proposto por Savi et al. (2010), as teorias de avaliação utilizadas estão descritas a seguir.

\subsection{O modelo de Avaliação de Treinamento de Kirkpatrick}

Donald Kirkpatrick desenvolveu um modelo de avaliação de treinamento baseado em 4 níveis. Segundo Garcia (2000), "sua estrutura auxilia na tomada de decisão e na estruturação da avaliação das atividades de aprendizado". Os 4 níveis do modelo de

\footnotetext{
${ }^{1}$ Site da Yoyo Game: https://www.yoyogames.com/studio
} 
avaliação de treinamentos proposto por Kirkpatrick (1994) são: (1) reação; (2) aprendizagem; (3) comportamento e (4) resultados.

O modelo proposto por Savi et al. (2010) utiliza apenas o nível 1 do modelo de avaliação de treinamentos de Kirkpatrick (1994), pois este é o que mais se enquadra na avaliação de jogos, tendo por finalidade identificar a reação dos usuários durante a utilização do jogo. Savi et al. (2010) define que o processo de avaliação da reação dos alunos é de suma importância, pois indica o quão o software de treinamento se fez eficiente, possibilitando possíveis melhorias. Dessa forma, é necessário que os alunos reajam favoravelmente a ele. Do contrário, os mesmos não se sentirão motivados a aprender.

\subsection{Taxonomia de Bloom}

Segundo Jesus e Haabe (2009), Benjamim S. Bloom criou na década de 50 a taxonomia, com o objetivo de facilitar a troca de questões entre professores de diversas faculdades. Cada questão avaliava o mesmo objetivo de aprendizagem. A taxonomia de Bloom é composta por seis categorias do domínio cognitivo: conhecimento, compreensão, aplicação, análise, síntese e avaliação. Porém, o método de avaliação proposto por Savi et al. (2010) utiliza apenas os 3 primeiros níveis, pois os demais são vistos como complexos para serem utilizados na avaliação de jogos. A Taxonomia de Bloom não fornece um método de avaliação completo, porém a sua utilização no processo de treinamento, permite levantar questões para identificar os principais desafios relacionados ao problema de pesquisa. Pois a taxonomia ao ser organizada de forma hierárquica permite a organização dos resultados, definindo uma linguagem comum no compartilhamento dos mesmos.

Atualmente, a Taxonomia de Bloom é utilizada por pesquisadores para medir os resultados e comparar tudo a partir de programas de métodos de aprendizagem. Várias modificações da Taxonomia de Bloom têm sido propostas, porém a descrição de Bloom de domínios de aprendizagem e níveis de complexidade ainda é largamente utilizado (MUNZENMAIER, 2013). Ferraz (2010) apresenta as 3 categorias da Taxonomia de Bloom utilizadas neste trabalho, são elas: (1) conhecimento: habilidade de utilizar conhecimentos prévios; (2) compreensão: habilidade de compreender e dar significado ao conteúdo; (3) aplicação: habilidade de utilizar informações em novas situações concretas.

\subsection{Modelo ARCS}

A motivação é um componente importante para que determinados objetivos sejam alcançados, trata-se de um elemento capaz de impulsionar as ações dos indivíduos. Moraes (2007) explica que a motivação é algo visceral, ou seja, um sentimento, ou se tem ou não se tem, isso não quer dizer que não seja possível fazer nada para que as pessoas possam vivenciá-la. No ambiente educacional, a motivação é vista como um sentimento voluntário, o qual deverá ser tratado como o desejo de continuar aprendendo.

Dentre os métodos utilizados para desenvolver táticas de motivação no processo de ensino e aprendizagem, destaca-se o modelo ARCS. Keller (2004) apresenta o modelo ARCS como um método capaz de fornecer ao aluno confiança em relação às expectativas e resultados esperados. Bem como fornece ao professor a possibilidade de 
administrar os resultados intrínsecos e extrínsecos dos alunos. Este modelo classifica o processo de motivação em 4 categorias: (1) atenção: trata-se de manter um nível considerável de atenção do aluno durante o processo de aprendizagem; (2) relevância: O aluno deverá relacionar o conteúdo abordado com os seus objetivos enquanto aluno e com o seu futuro profissional; (3) confiança: o aluno deverá se sentir confiante nas suas próprias ações durante o processo de aprendizagem; (4) satisfação: os alunos necessitam ter sentimentos positivos após a experiência de aprendizagem.

\subsection{User Experience em jogos}

Savi et al. (2010) define o conceito de User Experience (UX) como sendo uma área que aborda as respostas de uma pessoa em relação ao uso de um produto, sistema ou serviço. Estes produtos provocam experiências nos usuários. Ele ressalta que é objetivo da UX avaliar o entendimento dessas experiências que as pessoas têm com os produtos. O modelo proposto por Savi et al. (2010) levou em consideração quatro métodos de avaliação de jogos encontrados na literatura que levam em consideração o conceito de experiência do usuário (POELS, KORT e IJSSELSTEIJN, 2007; JENNETT et al. 2008; FU, SU e YU, 2009; GÁMEZ, 2009). Dos modelos avaliados, foram retirados os conceitos que mais se repetiram.

Desta forma, 6 categorias foram elaboradas: (1) imersão: trata-se do nível de envolvimento que o jogador tem com o jogo; (2) interação social: está relacionado com $\mathrm{o}$ ato de compartilhar as experiências do jogo com os demais usuários; (3) desafio: $\mathrm{O}$ jogo deve fornecer ao aluno um ambiente desafiador, estando adequado ao nível de habilidade do jogador; (4) diversão: o jogo deverá proporcionar ao aluno satisfação, relaxamento e diversão; (5) controle: o jogo deve fornecer ao usuário o controle sobre as suas ações; (6) competência: o aluno deverá desenvolver suas habilidades para acompanhar o desenrolar do jogo, dessa forma exercer sua competência ao ultrapassar os desafios impostos ao mesmo.

\section{Resultados}

\subsection{Jogo Eletrônico Educativo - NiceTown}

Como resultado, foi desenvolvido um jogo educativo com o intuito de abordar parte do conteúdo de educação ambiental, presente nos currículos do ensino fundamental. A proposta de desenvolvimento foi analisada por professores de Computação, Psicologia da Aprendizagem, de Ciências e por alunos do ensino fundamental. Os quais contribuíram com sugestões e críticas em relação a aspectos técnicos, de conteúdo e roteiro do jogo.

O jogo, denominado de NiceTown, fornece o conteúdo de forma lúdica e atrativa retratando problemas que afetam o meio ambiente e o dia-a-dia das pessoas. NiceTown tem como cenário uma cidade fictícia, onde o objetivo do aluno é basicamente resolver os problemas que a afetam. Para isso, o jogador terá que observar a situação da cidade, refletir sobre quais ações devem ser realizadas e agir para alcançar os objetivos com eficiência. Na Figura 1, é mostrada a tela inicial e o menu Ajuda para orientar o jogador sobre os procedimentos e usabilidade.

NiceTown possui dois estágios: Coleta Seletiva e Saneamento Básico. O excesso de lixo, assim como a falta de saneamento básico provoca impactos ambientais e 
prejudica a vida dos moradores de NiceTown. Por se tratar de um jogo não-linear, o usuário tem a liberdade de escolher entre os dois estágios, a partir da interpretação do nível de satisfação da cidade.

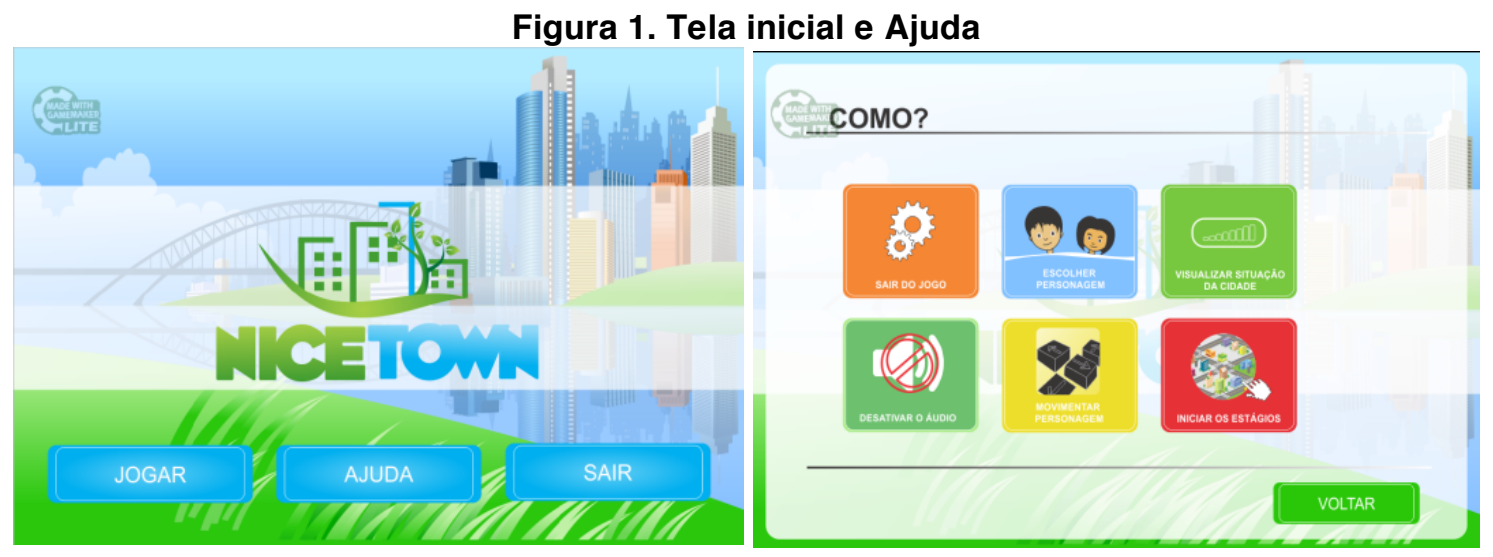

Como pode ser observado na Figura 2, este nível é composto pela situação do lixo e do saneamento básico, que tem como escala péssimo, ruim, bom e ótimo. $\mathrm{O}$ usuário deve tomar a decisão correta para a cidade, e assim, deixá-la mais agradável para seus habitantes. Alguns prédios são interativos, assim como a padaria, banca de revista, mercado, escola e prefeitura. Na banca de revista, notícias são atualizadas automaticamente de acordo com a situação da cidade. Na escola, simula-se uma aula em que uma professora ensina os conceitos básicos abordados no jogo, as lixeiras corretas para depositar cada tipo de lixo e benefícios da correta utilização do lixo reciclado e esgoto tratado.

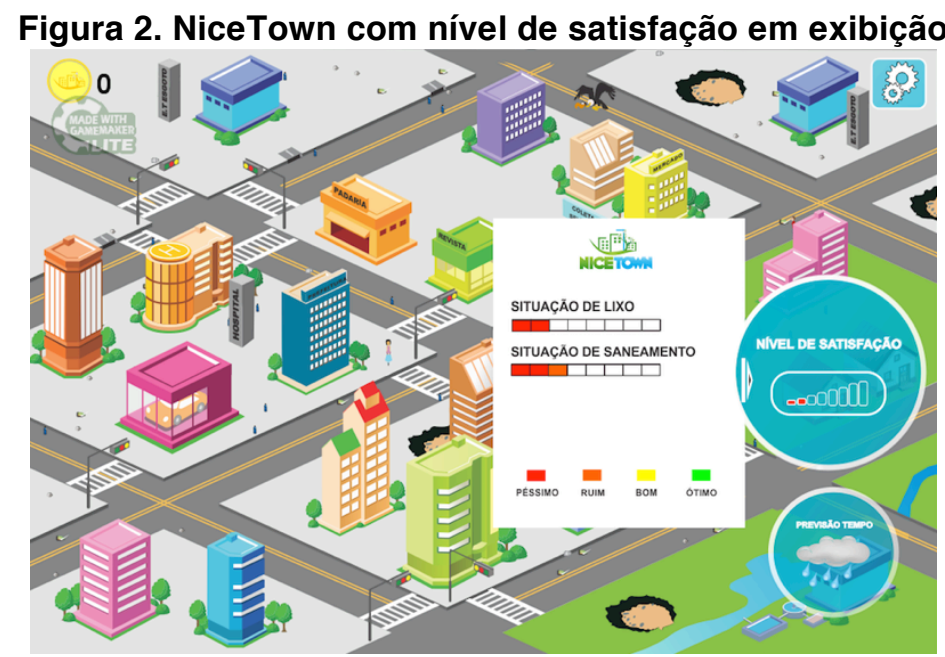

No mercado, o jogador pode comprar recursos para auxiliar na coleta do lixo. Para isso, foi criada a moeda NiceCoin. A cada lixo coletado corretamente, de acordo com as cores das lixeiras e tipos de lixo, o jogador ganha 2 NiceCoins. Esses recursos são: Cesta de mão - custa 40 Nicecoins e suporta até 4 itens de lixo; Carrinho de mão custa 70 Nicecoins e suporta até 8 itens de lixo; Lixeira - custa 90 Nicecoins e suporta até 12 itens de lixo. Além desse metodo, após completar cada nível do estágio de Saneamento Básico, o jogador ganha 8 NiceCoins. Desta forma, estimula o jogador a depositar os lixos e realizar o saneamento corretamente. 
Para iniciar o estágio de saneamento básico, o aluno deve procurar uma estação de tratamento de esgoto, tendo em vista que a cidade possui 3 estações. Após entrar em uma das três estações, o jogador visualizará um desafio como exibido na Figura 3. Uma vez iniciado, o usuário terá 1 minuto para encaixar os canos corretamente, de modo a montar uma passagem para o esgoto. Salienta-se que o nível de dificuldade aumenta conforme o jogador conclui cada uma das estações de tratamento de esgoto. Para iniciar o estágio da coleta seletiva, o usuário deve procurar o ponto de coleta seletiva. Em seguida, terá também o tempo de 1 minuto para recolher os lixos espalhados pela cidade, depositando-os nas lixeiras correspondentes, conforme demonstrado na Figura 4. Ao final de cada tempo, o jogador é parabenizado pelo que conseguiu fazer e apresenta-se para ele o que a cidade ganhou com o feito dele.

Figura 3. Estágio Saneamento Básico

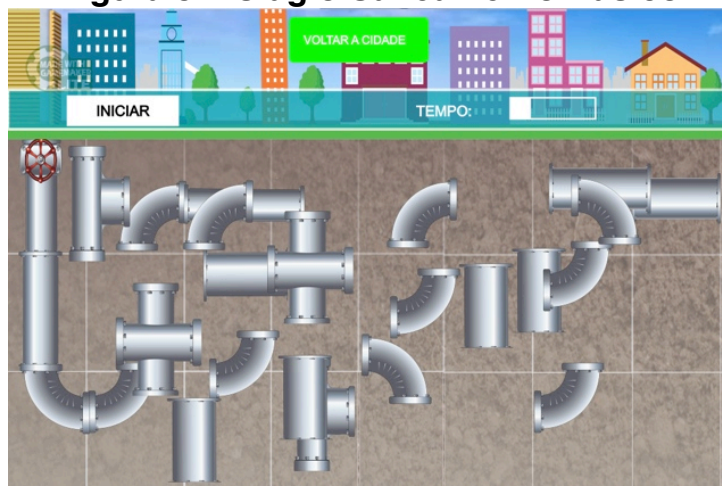

Figura 4. Estágio Coleta Seletiva

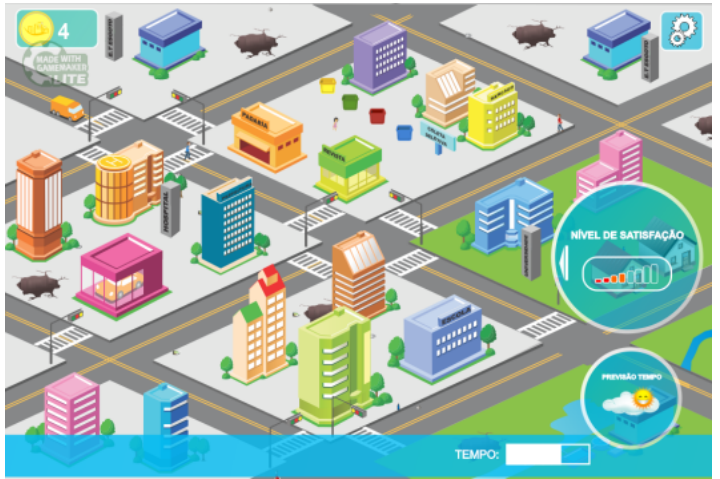

\subsection{Avaliação do Jogo NiceTown}

Como mencionado na seção anterior, foi utilizado como base o questionário proposto por Savi et al. (2010) para avaliação do jogo NiceTown com os 40 alunos do $6^{\circ}$ ano do Ensino Fundamental da Escola de Aplicação Professora Ivonita Alves Guerra. Este questionário tem como objetivo avaliar os seguintes tópicos: Motivação, Experiência do usuário e Conhecimento. Para cada categoria são apresentadas afirmações para que o jogador classifique se concorda, não concorda ou não sabe responder. As afirmações referentes ao tópico Motivação são apresentadas na Tabela 1.

Tabela 1. Afirmativas do Tópico Motivação

\begin{tabular}{|c|l|}
\hline Categorias & \multicolumn{1}{c|}{ Afirmativas } \\
\hline Atenção & $\begin{array}{l}\text { (1) Houve algo interessante no início e durante o jogo que capturou minha atenção. } \\
\text { (2) O design de interface do jogo é atraente. }\end{array}$ \\
\hline Relevância & $\begin{array}{l}\text { (3) O conteúdo do jogo está relacionado com as coisas que eu já sabia. } \\
\text { (4) Eu gostei do jogo ao ponto de desejar aprender mais sobre os conteúdos abordados. } \\
\text { (5) O conteúdo do jogo é relevante para meus interesses. }\end{array}$ \\
\hline Confiança & (6) O jogo foi mais difícil de entender do que eu gostaria que fosse. \\
\hline Satisfação & (7) Eu me senti bem quando terminei o jogo. \\
\hline
\end{tabular}

A Tabela 2 demonstra as respostas referentes às afirmativas do tópico Motivação. De acordo com os resultados, pode-se perceber que todos os alunos acharam algo interessante no jogo e que a grande maioria não sentiu desmotivação ao jogar. Grande parte dos alunos já tinha algum conhecimento sobre os conteúdos abordados e que ficaram interessados em aprender mais sobre o assunto. Constatou-se que o jogo foi considerado de fácil entendimento e que eles se sentiam bem ao terminar o jogo. 
Tabela 2. Respostas do Tópico Motivação

\begin{tabular}{|c|c|c|c|c|c|c|c|}
\hline & \multicolumn{2}{|c|}{ Atenção } & \multicolumn{3}{|c|}{ Relevância } & Confiança & Satisfação \\
\hline Questões & $\mathbf{( 1 )}$ & $\mathbf{( 2 )}$ & $\mathbf{( 3 )}$ & $\mathbf{( 4 )}$ & $\mathbf{( 5 )}$ & $\mathbf{( 6 )}$ & $\mathbf{( 7 )}$ \\
\hline Concordo & 40 & 38 & 28 & 36 & 30 & 0 & 35 \\
\hline Não Sei & 0 & 2 & 7 & 4 & 9 & 6 & 5 \\
\hline Discordo & 0 & 0 & 5 & 0 & 1 & 32 & 0 \\
\hline
\end{tabular}

As afirmativas referentes ao tópico Experiência do Usuário são apresentadas na Tabela 3 .

Tabela 3. Afirmativas do Tópico Experiência do Usuário

\begin{tabular}{|c|l|}
\hline Categorias & \multicolumn{1}{c|}{ Afirmativas } \\
\hline Imersão & (8) Eu não percebi o tempo passar enquanto jogava. \\
& (9) Eu perdi a consciência do que estava ao meu redor enquanto jogava. \\
\hline Desafio & $\begin{array}{l}\text { (10) Eu me senti ansioso e entediado durante o jogo. } \\
\text { (11) O jogo me manteve motivado a continuar utilizando-o. } \\
\text { (12) O meu conhecimento foi gradualmente aumentado com a superação dos desafios } \\
\text { propostos no jogo. }\end{array}$ \\
\hline Habilidade e & $\begin{array}{l}\text { (13) Eu senti que o jogo era apropriado para o meu nível de aprendizagem. } \\
\text { Competência }\end{array}$ \\
\hline Diversão & $\begin{array}{l}\text { (15) Senti que estava tendo progresso durante o desenrolar do jogo. } \\
\text { (16) Eosto de utilizar este jogo por bastante tempo. } \\
\end{array}$ \\
\hline
\end{tabular}

De acordo com a Tabela 4, pode-se observar o entusiasmo dos alunos perante o jogo e a animação que os mantêm focados. Além disso, afirmam em sua maioria que o jogo está de acordo com o nível de aprendizagem e que ficaram desapontados quando chegaram ao fim do jogo.

Tabela 4. Respostas do Tópico Experiência do Usuário

\begin{tabular}{|c|c|c|c|c|c|c|c|c|c|c|}
\hline & \multicolumn{3}{|c|}{ Imersão } & \multicolumn{3}{|c|}{ Desafio } & \multicolumn{3}{c|}{ Habilidade } & \multicolumn{3}{c|}{ Diversão } \\
\hline Questões & $\mathbf{( 8 )}$ & $\mathbf{( 9 )}$ & $\mathbf{( 1 0 )}$ & $\mathbf{( 1 1 )}$ & $\mathbf{( 1 2 )}$ & $\mathbf{( 1 3 )}$ & $\mathbf{( 1 4 )}$ & $\mathbf{( 1 5 )}$ & $\mathbf{( 1 6 )}$ & $\mathbf{( 1 7 )}$ \\
\hline Concordo & 40 & 25 & 0 & 29 & 31 & 37 & 31 & 33 & 37 & 40 \\
\hline Não Sei & 0 & 13 & 5 & 7 & 8 & 3 & 4 & 5 & 3 & 0 \\
\hline Discordo & 0 & 2 & 35 & 4 & 1 & 0 & 5 & 2 & 0 & 0 \\
\hline
\end{tabular}

As afirmativas do Tópico Conhecimento são apresentadas na Tabela 5.

Tabela 5. Afirmativas do Tópico Conhecimento

\begin{tabular}{|c|l|}
\hline Categorias & \multicolumn{1}{|c|}{ Afirmativas } \\
\hline Conhecimento & $\begin{array}{l}\text { (18) Depois do jogo consigo lembrar das várias informações relacionadas } \\
\text { ao tema apresentado no jogo. }\end{array}$ \\
\hline Compreensão & $\begin{array}{l}\text { (19) Depois do jogo consigo compreender melhor os assuntos } \\
\text { apresentados no jogo. }\end{array}$ \\
\hline Aplicação & $\begin{array}{l}\text { (20) Depois do jogo sinto que consigo aplicar melhor os temas } \\
\text { relacionados com o jogo. }\end{array}$ \\
\hline
\end{tabular}

De acordo com a Tabela 6, a maioria dos alunos compreendeu e se lembram do que aprendeu, tornando-os cidadãos conscientes sobre a Educação Ambiental. Além disso, percebe-se que eles se consideram capazes de colocar em prática o que aprenderam. Tal fato deve-se também a exposição de exemplos de aplicações que o jogo apresentava ao final de cada desafio, seja na coleta seletiva, como também no senamento básico.

Tabela 6. Respostas do Tópico Conhecimento

\begin{tabular}{|c|c|c|c|}
\hline & Conhecimento & Compreensão & Aplicação Prática \\
\hline Questões & $\mathbf{( 1 8 )}$ & $\mathbf{( 1 9 )}$ & $(\mathbf{2 0 )}$ \\
\hline Concordo & 38 & 29 & 38 \\
\hline Não Sei & 2 & 3 & 2 \\
\hline Discordo & 0 & 8 & 0 \\
\hline
\end{tabular}




\section{Considerações Finais}

Os jogos eletrônicos educativos têm se tornado cada vez mais presentes, como uma ferramenta lúdica no processo de ensino e aprendizagem. Como principais contribuições, este trabalho visou apresentar o desenvolvimento e a avaliação de um jogo voltado para o ensino de Educação Ambiental, com foco em Coleta Seletiva e Saneamento Básico, desenvolvido por alunos do curso de Licenciatura em Computação durante a disciplina Estágio Supervisionado III.

Com a intenção de obter sugestões e críticas de melhorias, o jogo foi desenvolvido em cooperação com professores de Computação, Psicologia da Aprendizagem e de Ciências. Utilizou-se o processo Iterativo e Incremental de desenvolvimento de software para auxiliar na construção de um jogo que atendesse os aspectos lúdicos e pedagógicos. Ao longo das iterações, era visível o entusiasmo demonstrado pelos alunos e pelo professor de Ciências. Segundo este professor, "este jogo facilitará e muito minhas aulas. Principalmente para colocar em prática aquilo que ministro em sala. Com esta ferramenta, os alunos se sentirão bem mais motivados a participar".

Após terminado o desenvolvimento, uma turma com 40 alunos do $6^{\circ}$ ano do Ensino Fundamental (público-alvo do jogo) testou o jogo e em seguida cada um respondeu a um questionário de avaliação baseado no modelo de Savi et al. (2010). Conforme apresentado na seção anterior, observou-se que a grande maioria dos alunos aprovaram o jogo sob os tópicos Motivação, Experiência do usuário e Conhecimento.

Como trabalhos futuros, pretende-se aplicar este jogo para avaliação em outras escolas e obter um maior número de feedbacks com outros professores de Ciências. Além disso, pretende-se desenvolver outros problemas ambientais para agregar ao jogo, tais como, desmatamento e esfeito estufa. Desta forma, tem-se a possibilidade de fortalecer o processo de ensino e aprendizagem na área de Educação Ambiental.

\section{Referências}

Bloom, B. (1956). "Taxonomy of educational objectives: The classification of educational goals: Handbook I". Cognitive domain. New York; Toronto: Longmans, Green.

Ferraz, A. P. C. M; BELHOT, R. V. (2010). "Taxonomia de Bloom: revisão teórica e apresentação das adequações do instrumento para definição de objetivos instrucionais". Gest. Prod, São Carlos.

Fu, F.; SU, R.; YU, S. (2009). "EGameFlow: A scale to measure learners' enjoyment of e-learning games”. Comput. Educ., , v. 52, n. 1, p. 101-112.

Gámez, E. H. C. (2009). "On the Core Elements of the Experience of Playing Video Games". Tese de doutorado - UCL Interaction Centre Department of Computer Science.

Garcia, A. F; CUNHA, L. M. (2000) Avaliação em Instrução Baseada na WEB. PUC.

Gomes, C. J. S. (2006). “O Aluno como Autor de Jogos Educacionais em Computador”. Universidade Federal do Rio Grande do Sul, Centro Interdisciplinar de Novas Tecnologias na Educação. Porto Alegre. 
Gomes, P. (2012). “As 12 tendências da educação brasileira até 2017”. Disponível em: $<$ http://porvir.org/porpensar/12-tendencias-da-educacao-brasileira-ate2017/20121123>. Acessado em 06/06/2014.

Jennett, C.; Cox, A. Cairns, P.; Dhoparee, S.; Epps, A.; Tijs, T.; Walton, A. (2008). "Measuring and defining the experience of immersion in games". Int. J. Hum. Comput. Stud., v. 66, n. 9.

Jesus, E. A; Raabe, A. L. A. (2009). "Interpretação da Taxonomia de Bloom no Contexto da Programação Introdutória". Universidade do Vale do Itajaí - UNIVALI, XX Simpósio Brasileiro de Informática na Educação,.

Keller, J. M.; Suzuki, K. (2004). "Learner Motivation and E-Learning Design: A Multinationally Validated Process". Journal of Educational Media, v. 29, n. 3, p. 229-239, Outubro.

Kirkpatrick, D. (1994). "Evaluating Training Programs - The Four Levels". BerrettKoehlerPublishers, Inc.

Malaquias, M.; Ribeiro, S. (2013). “A importância do lúdico no Processo de EnsinoAprendizagem no Desenvolvimento da Infância". Disponível em: $<$ http://psicologado.com/atuacao/psicologia-escolar/a-importancia-do-ludico-noprocesso-de-ensino-aprendizagem-no-desenvolvimento-da-infancia>. Acesso em: 25 de março de 2014.

Melo, M. J. (2009). “Oceano verde - uma práxis de educação ambiental: análise da experiência desenvolvida numa escola pública de ensino médio de Brasília”. $245 \mathrm{f}$. Dissertação (Mestrado em Educação) - Universidade de Brasília. Brasília.

Ministério da Educação. (2009) O que Fazem as Escolas que Dizem que Fazem Educação Ambiental? Coleção Educação para todos, Brasília.

Munzenmaier, C; Rubin, N. (2013). "BLOOM'S TAXONOMY: What's Old Is New Again”. The eLearning Guild, Santa Rosa.

Pietruchinsk, H. M; NETO, J.C.; Malucelli, A; Reinehr, S. (2011) "Os jogos educativos no contexto do SBIE: uma revisão sistemática de Literatura", In: XXII Simpósio Brasileiro de Informática na Educação (SBIE), Aracaju - SE, Brasil. pág.: 476-485.

Poels, K.; Kort, Y. D.; Ijsselsteijn, W. (2007). “It is always a lot of fun!": exploring dimensions of digital game experience using focus group methodology In: PROCEEDINGS OF THE 2007 CONFERENCE ON FUTURE PLAY. Toronto, Canada: ACM, 2007.

Pressman, R. S. (2006). "Engenharia de Software". $6^{\circ}$ ed. Rio de Janeiro: McGraw-Hill.

Reis, T. (2014). "A Diferença entre o Plano de Projeto e o Project Model Canvas". Disponível em <http://www.projectbuilder.com.br/blog-pb/entry/blog-gestao-deprojetos/a-diferenca-entre-o-plano-de-projeto-e-o-project-model-canvas $>$. Acesso em: 01 de Jul de 2014.

Savi, R.; Von Wangenheim, C.G.; Ulbricht, V.; Vanzin, T. (2010). "Proposta de um modelo de avaliação de jogos educacionais". Novas Tecnologias na Educação, v. 8, n.3, dezembro. 